\title{
Una crónica no anunciada: El «otro» latino en el ámbito teatral de Estados Unidos
}

\author{
An Unexpected Chronicle: The «Other» Latino \\ Theatre in the U.S.
}

\author{
Beatriz J. Rizk \\ Miami-Dade College, Prometeo Theatre. Miami, U.S. \\ BJRizk@aol.com
}

1

Resumen - Este ensayo se concentra en la contribución al arte escénico de las comunidades latinas en Estados Unidos de miembros de «otros» grupos nacionales (venezolanos, dominicanos, peruanos, argentinos, etc.) que están rompiendo con los patrones impuestos por la producción de los tres grupos mayoritarios: los méxico-americanos, los puertorriqueños y los cubano-americanos. La producción artística de este "nuevo" grupo inmigratorio ocupa un "espacio sensorial» en el que diferentes gramáticas, representando algunas veces disímiles indicadores culturales, se encuentran entreverados de manera yuxtaposicional. El intentar rastrear una genealogía geográfica se problematiza pues es obvio que no se puede hablar, entre otras cosas, de "discontinuidades culturales». Las manifestaciones teatrales de este "otro" grupo son constitutivas de un canon alternativo, que reta cualquier lectura simplista o esfuerzo de clasificación en una producción nacional cerrada, dando paso a un acercamiento crítico, como el que intentamos aquí, más «globalizado».

Palabras clave: Teatro latino/latinoamericano, Estados Unidos, arte escénico.

Abstract - This essay focuses on the contribution to the Latino performing arts, in the United States, by members of "other» national groups (Venezuelans, Dominicans, Peruvians, Argentines and so on) who are breaking away from established patterns set by the production of the three major immigration groups: the Mexican-Americans, Puerto Ricans and Cuban-Americans. The artistic productions of this «new» immigration group occupy a «sensorial space» where different grammars, representing some times dissimilar cultural markers, are intertwined in a juxtapositional manner. Mapping a geographic genealogy becomes complicated as it is evident that one cannot talk about «cultural discontinuities» among other things. Their work is constitutive of an alternative canon, which defies any simplistic reading or efforts to classify them into a bounded national production, giving way, as we intent to do here, to a more «globalized» critical approach.

Keywords: Latino/Latin American Theatre, United States, performing art. 
Aunque la presencia en Estados Unidos de las comunidades latinas se deba a la conquista, colonización, inmigración, o exilio voluntario o forzoso, todos sus miembros se enfrentan y comparten los mismos problemas, la misma discriminación y, obvio, los mismos éxitos. La diferencia es realmente de gradación, no de opción. Es, de hecho, una comunidad que revive su historia de manera continua y activa sus símbolos históricos para encontrar los nexos que puedan brindar un sistema de soporte a tan heterogénea composición. Contrario a la opinión pública generalizada, la(s) comunidad(es) conforma(n) un grupo con tantas subdivisiones étnicas como existen en el mundo más allá de sus fronteras impuestas o metafóricas. En este sentido, no puede ser dividida en términos de blanco y negro, como los estadounidenses de origen anglo-sajón tradicionalmente perciben su sociedad y por extensión a todas las demás. Blancos y negros, y gente cuyo origen proviene de los pueblos indígenas, de Asia, del Medio Oriente, etc., constituyen la comunidad. Esta mezcla heterogénea refleja su bagaje cultural. Es más, aun la noción de una región cultural dentro de la comunidad ya no se aplica a ciudades como Nueva York, o Miami, y otros muchos lugares del país. Los enclaves que se asumían hace treinta años como predominantemente chicanos, ${ }^{1}$ puertorriqueños, o cubanos, ya no pueden ser definidos en términos esencialistas o como pertenecientes a una cultura específica. No hace mucho, la crítica Susana Chávez-Silverman señalaba, refiriéndose al poema «Kyrie Eleison for La Llorona» de Alicia Gaspar de Alva, que:

If La Llorona «lives» in such desperate and quintessentially American places as Iowa City, Boston, San Francisco, and the Southwest, it is because we are there too. We see and hear her. At the same time that these lines problematize «the» border as the (appropriate)? Homeland for Chicanos, [...] [the poet] makes manifest the «Latinization of America» as a given fact (2003: 221).

Durante las últimas décadas, por otra parte, la creciente globalización económica ha impulsado más inmigración transnacional; individuos provenientes de todas las Américas han llegado a reforzar nuestras tradiciones, nuestro sentido de la identidad y nuestra afiliación a ese mundo que está situado al sur del Río Grande. Ya hay una extensa bibliografía, incluyendo en el campo del teatro, sobre los tres grupos más grandes dentro de la comunidad, referidos antes, quienes representan el $60 \%$, el $14 \%$ y el $6 \%$ de la misma, respectivamente, en los que no entraremos aquí; de ahí el énfasis en el "otro" latino del título de este ensayo. ${ }^{2}$ Vamos a concentrarnos aquí en las contribuciones de los

1 El término "chicano/chicana» se aplica al méxico-americano/a pero tiene una connotación política derivada de su involucración en el llamado Movimiento Chicano contra-cultural de los años sesenta y setenta en Estados Unidos. Los individuos que se identifican como tales asumen una posición contestataria social y política ante la cultura dominante. Dicho sea de paso, es obvio que no todos los/as méxico-americanos/ as se identifican como chicanos/as. Para mayor información sobre el desarrollo político del Movimiento, ver el excelente estudio de Arturo F. Rosales, Chicano: The History of the Mexican American Civil Rights Movement (1997).

2 Sobre el teatro, que es la materia que nos interesa aquí, ver, en el caso de los chicano/mexico-americanos, los libros seminales tanto de Nicolás Kanellos (1993 y 1990), como los de Jorge Huerta (1982 y 2001). En cuanto el teatro puertorriqueño en Estados Unidos quizás la compilación de ensayos más completa que se ha hecho hasta ahora la llevó a cabo el Ateneo Puertorriqueño, bajo la iniciativa del dramaturgo Roberto Ramos-Perea (2006). Sobre el teatro cubano-americano habría que buscar en los prólogos a las varias antologías de teatro que se han publicado como las de Rodolfo J. Cortina (1991) de Luis F. González-Cruz y Francesca M. Colecchia (1992) y de Lilian Manzor y Alberto Sarraín (2005). Ver también los ensayos de José Escarpanter (1988) y de Beatriz J. Rizk (2000). 
teatristas latinos que provienen de otros grupos, como son los dominicanos, venezolanos, peruanos, etc., cuyas voces están siendo escuchadas en el país del norte, desafiando estereotipos y rompiendo con esquemas limitadores. Este grupo representa un $20 \%$ de la población latina en el país y a pesar de su tardía presencia en el panorama nacional es bastante visible pues está siendo proyectado prioritariamente en los exitosos medios de comunicación latinos. ${ }^{3}$ Ahora, si el impacto de su acrecentada presencia, debido al deterioro económico que ha sacudido a varios países latinoamericanos, sobre todo después del advenimiento de las reformas neoliberales a partir de la década de los ochenta, ha sido omitida por la mayoría de las investigaciones, ya sean hechas desde una perspectiva demográfica, histórica, antropológica o cultural, en lo referente a Estados Unidos, por lo menos su llegada sí ha sido registrada por estudiosos latinoamericanistas:

Sizable immigrant communities from countries that exported few or non migrants prior to the 1990s have emerged in the United States. These include Brazil (Levitt, 2001); Ecuador (Kyle, 2000); and Peru (Boswell and Skop, 1995). Several recent reports from the sending countries indicate mass departures to the United States, Spain, and Italy from Argentina, Colombia, Ecuador, Peru, and the Dominican Republic (Latin American Weekly Report 2002, 56-57; Criado 2001; Itzigsohn 2000; Guarnizo, Sánchez, and Roach 1999) (Portes y Hoffman 2003: 70-1). ${ }^{4}$

Por otra parte, este grupo está, en su gran mayoría, conformado por miembros de la clase media o sus segmentos empobrecidos, lo que hace que sus valores sociales estén firmemente anclados en los de su misma clase. Por lo tanto, rehúsan, por ejemplo, el sistema de códigos raciales usado en los Estados Unidos, conservando sus propias convicciones culturales tradicionales. Desde esta perspectiva, el grado de «blanqueamiento» o "negritud» con que un individuo se identifique no tiene nada que ver con el nivel de «latinidad» con el que se asocie. Las categorías raciales en América Latina, en no pocas instancias, se esconden detrás de un cuadro social estratificado. Aunque muchos inmigrantes latinoamericanos reconocen, de hecho, el «mestizaje» cultural como base de sus propias culturas, y hasta llegan a hacer suyos los prejuicios raciales y discriminación étnica vigentes en la sociedad receptora, la mayoría no acepta la radical y tradicional división entre blanco y negro de la sociedad norteamericana. ${ }^{5}$ En América Latina, como

3 Nos referimos a las económicamente poderosas cadenas nacionales de televisión latinas como Univisión y Telemundo, al sistema de cable Galavisión y aun a la cadena Televisa de México, Venevisión de Venezuela, Radio Cadena Nacional de Colombia y Globo del Brasil, las que están compartiendo parte del mercado norteamericano a través de las entidades mencionadas antes.

4 Para mayor información sobre el tema revisar las publicaciones a las que se refieren Portes y Hoffman: Thomas D. Boswell y Emily Skop, Hispanic National Groups in Metropolitan Miami (Miami: Cuban American National Council, 1995); María Jesús Criado, La línea quebrada: historias de vida de migrantes (Madrid: Consejo Económico y Social, Instituto Universitario Ortega y Gassett, 2001); Luis E. Guarnizo, Arturo I. Sánchez y Elizabeth M. Roach, «Mistrust, Fragmented Solidarity, and Transnacional Migration: Colombians in New York and Los Angeles", Ethnic and Racial Studies 22 (1999): 367-96; José Itzigsohn, Developing Poverty: The State, Labor Market Deregulation, and the Informal Economy in Costa Rica and the Dominican Republic (University Park, PA: Pennsylvania State University Press, 2000); David Kyle, Transnational Peasants: Migration, Networks, and Ethnicity in Andean Ecuador (Baltimore, MD: Johns Hopkins Univ. Press, 2000); Peggy Levitt, The Transnational Villagers (Berkely: University of California Press, 2001).

5 De manera por demás curiosa, mientras escribimos estas líneas (julio de 2008), ante el posible acceso a la presidencia de Estados Unidos de un afro-descendiente, el senador Barack Obama, son varios los observadores e historiadores de la nación que públicamente han declarado que no creen que este hecho, sin precedente en la historia del país, cambie o modifique realmente las desniveladas relaciones raciales y la manera como se percibe la gente. 
sabemos, hay una miríada de nombres para definir la gradación entre estos dos polos extremos, además de que, como dice el viejo refrán: "negro con plata es blanco». Esto no quiere decir que la negación del ancestro negro, por ejemplo, no sea mayor en América Latina, incluido el Caribe, que entre los latinos/latinas en Estados Unidos, como algunos investigadores ya lo han señalado (Jiménez-Muñoz, 1993: 11). Un ejemplo pertinente nos lo suministra el censo oficial de Puerto Rico, en el 2000 , cuando solo un $4 \%$ de la población se registró como negro, o afro-descendiente. En cuanto al idioma, muchos usan el español como el lenguaje principal de comunicación, estableciendo su valor no solamente como instrumento público sino también como un vehículo apto para desarrollar discursos que tengan implicaciones políticas y sociales a la par con el inglés. Ahora, aquellos que escriben en inglés, "pero su cultura y su identidad nacional residen en otra parte», como señala el crítico Román de la Campa, pertenecen a un grupo que está socavando como nunca antes se ha hecho «la relación entre lenguaje, literatura y nación», especialmente hoy en día cuando el inglés se está convirtiendo en «la lingua franca de la globalización» (2005: 302).

Desde el punto de vista del tan debatido terreno de la identidad latina, ${ }^{6}$ particularmente ahora que la alternativa cultural está moviéndose de la estricta resistencia a la asimilación hacia un concepto más elaborado de la frontera como sitio en proceso de expansión en el que las culturas se cruzan e interfieren libremente, ya se incluye el llamado físicamente crossing-over (o sea el pasar de una cultura a otra), o el viajar constantemente al país de origen. ${ }^{7}$ Este fluir entre el aquí y el allá, para los dramaturgos estudiados aquí, ha dejado de ser un hecho aislado para convertirse en un hábito natural. Estamos, por lo tanto, yendo un paso más allá de los acostumbrados debates asociados con la identidad como estrategia versus la contra-identidad, como resistencia, que han servido de marco de referencia a casi toda la producción teatral latina en Estados Unidos, a favor de una ficción convincente de "nacionalismo» individual en el que «los diversos modos preformativos de mundos culturales e ideológicos bastante diferenciados derivados de una o más culturas encuentran su lugar ahí» (Shohat y Stam, 1994: 42).

En este sentido, el derraizamiento o la de/re/territorialización características de las historias de inmigración a duras penas pueden ser aplicadas aquí. Los dramaturgos que vamos a estudiar pertenecen a una comunidad transnacional que se siente contradictoriamente bien, y al mismo tiempo desplazada, en cualquier lugar donde se encuentre. Por otra parte, no lo apremia la necesidad imperiosa de asimilarse, o integrarse, en la sociedad receptora como fue el caso, por ejemplo, de los centroamericanos, en su mayoría campesinos desposeídos, que llegaron durante los años ochenta debido a la guerra civil que estaba asolando sus países de origen (el film El Norte (1983), de Gregorio Nava, nos

Un buen número de los estudios dedicados a la comunidad y no solo a sus expresiones artísticas sino también a la problemática social, parten de este punto que hasta ahora ha sido crucial: el de la asimilación o no asimilación a los patrones culturales anglo-sajones y el desafío a la llamada tesis de la «tercera generación». Según ésta, todos los grandes grupos de inmigrantes que han entrado al país, al llegar a la tercera generación han eliminado el idioma materno, las costumbres y tradiciones con las que llegaron al país. Es evidente que no ha sucedido este fenómeno en las comunidades latinas que persisten y han logrado conservar los mismos. Ver, entre otros, Edna Acosta-Belén y Barbara R. Sjostrom (1991); Frances R. Aparicio y Susana Chávez-Silverman (1997); Mary Romero, Pierrette Hondagneu-Sotelo y Wilma Ortiz (1997).

7 Hemos de señalar que la noción de la «frontera» como sitio del enunciado preferente de la producción literaria latina parte, en nuestro medio, del trabajo pionero de la lamentablemente desaparecida poeta e investigadora chicana Gloria Anzaldúa, en su libro seminal, Borderlands/La Frontera. The New Mestiza (1987). 
brinda un deplorable ejemplo del camino sin regreso que tomaron muchos de estos refugiados). Esta nueva ola inmigratoria, por el contrario, permanece bastante indiferente a las complicadas elaboraciones burocráticas de la sociedad norteamericana en su esfuerzo por clasificarlos. Por otra parte, aunque la identificación grupal no ha sido primordial para ellos, su complejidad empieza a revelarse en el momento en que se enfrentan con las tácticas discriminatorias raciales y sociales. De hecho, es a través del contacto con las tónicas racistas de la sociedad norteamericana, mientras viven y trabajan en el país, que algunos de ellos toman conciencia de su «entidad política» como latinoamericanos. La directora teatral de origen argentino Susana Tubert así lo expresa:

[...] I realized that although I have been an Argentinian all my life, it was through teaching and performing in the barrios that I have become a Latin American. It is ironic that I began to feel more Latin American in New York than I ever had in Argentina (2000: 327).

La dramaturga dominicana Luisa (Chiqui) Vicioso recuerda que fue cuando vivía en Nueva York, durante los años sesenta, que adoptó la identidad negra como un "gesto de solidaridad» con la militante Angela Davis. "Y desde entonces", comenta "siempre me he identificado con la Mujer Negra [,] tenía que descubrir primero que formaba parte de una cierta área geográfica y luego, que era latinoamericana» (en Horno-Delgado et al., 1989: 231). También funciona al revés, como parece le ocurrió a la autora Caridad Svich cuando encontró la esencia de lo que es un norteamericano en medio del núcleo de su familia cubana en el sur de la Florida: «Creo que fue en Miami en donde devine una verdadera norteamericana - una fusión de sensibilidades integradas en cuerpo y espíritu» (2000: 320).

Para otros, como el venezolano Gustavo Ott, quien pasa la mitad del año en San Petersburgo o Florida y la otra mitad en Caracas escribiendo sus obras desde los dos sitios, la subjetivización minoritaria a la que habitualmente son sometidos y devaluados los latinos en Estados Unidos es, como expresan algunos de sus personajes, una cuestión de «insuficientes fondos»; en otras palabras, y de acuerdo a los prejuicios vigentes de las sociedades latinoamericanas, una cuestión de estatus social. Algunas de sus obras tratan sobre la emigración venezolana en Estados Unidos. Un buen número entre estos inmigrantes, como en otras partes, se han mudado al norte en busca de mejores oportunidades como lo hicieron tres peloteros «muertos de hambre» en su obra 80 dientes, 4 metros y 200 kilos, ganadora del prestigioso premio Tirso de Molina: una historia sobre inmigración que se convierte en pesadilla a medida que la drogadicción, los enredos con la mafia y la violencia callejera se vuelven lugares comunes. Pero hay otros inmigrantes que vienen al país a estudiar, a buscar nuevos horizontes profesionales y/o a trabajar en su campo, sin que pese sobre sus cabezas el camino del no regreso. Este es el caso de la pareja matrimonial de Tu ternura Molotov (2003); Daniel es un abogado en proceso de ascender en el escalafón burocrático de su compañía y Victoria, una locutora de un canal de televisión local. ${ }^{8}$ Ocupados haciendo el amor, al comenzar la obra, con el claro y resuelto propósito de procrear, son interrumpidos por la llegada de un paquete dirigido a Victoria y al parecer entregado 12 años más tarde por el FBI. El paquete contiene una

Tu ternura Molotov ganó el primer premio en el Concurso de Dramaturgia Ricardo López Aranda en 2003 y tuvo su estreno en Palmas de Gran Canarias, en España, por el grupo Profetas de Mueble Bar en septiembre 2004. Desde entonces se ha montado en varios países de la América Latina y en Estados Unidos en donde hizo temporada en el Teatro Gala de Washington D.C., en 2007. 
mochila que le perteneció en la que se encuentran fotos suyas dando irrefutable evidencia de que tomó parte en un movimiento terrorista clandestino, gracias a un novio de la época, durante sus años de estudiante en Nueva York. Después de una tanda de recriminaciones por parte y parte, de las que el marido tampoco sale bien librado, el matrimonio al parecer sobrevive, como señala Emory Myslewsky, simplemente porque los dos son igualmente centrados en sí mismos y egoístas. ${ }^{9}$ Es, sin embargo, a través de sus diálogos, como cuando están eligiendo un nombre para el bebé que de manera desesperada están tratando de concebir, que sale a la superficie el «contexto" de la obra:

Victoria: [...] ¿Qué nombre le pondremos?

Daniel: Jesús Daniel, por ejemplo.

Victoria: ¿No es muy hispano?

Daniel: Somos hispanos.

Victoria: Pero tampoco hay que exagerar.

Daniel: Y no lo parecemos, es verdad.

Victoria: Yo paso en la calle por... por...

Daniel: ¿Y si es niña?

Victoria: Algo así como María Magdalena o Marie Magdalene.

Daniel: ¿Pero ésa no era una puta?

Victoria: Cállate, que ahora es santa.

Daniel: ¡Cómo cambian las cosas!

Victoria: Los grupos de presión y la «Correcte Politique».

Daniel: No me gusta.

Victoria: ¿Quizás María Esther?

Daniel: Judía.

Victoria: ¿Qué te parece Shalma?

Daniel: Muy negro.

Victoria: Y José.

Daniel: Suena a minoría.

Victoria: Somos una minoría.

Daniel: No seas estúpida. Minoría es alguien que no tiene dinero (89-90).

Esta nueva «inmigración» representada en la escena difícilmente puede encarnar una «subjetividad dividida» puesto que no tuvo lugar aquí una no-transculturación, o aculturación. Y poniéndolo en términos globalizantes, en muchas instancias, ya ha sido formada ideológicamente, para aceptar los valores y el estilo de vida norteamericanos, a través de la masiva difusión que los medios de comunicación y el cine norteamericanos tienen en el país en donde crecieron. De esta manera, las nociones pre-existentes que tengan sobre su identidad nacional no compiten con la sociedad receptora puesto que ésta ya ha informado de antemano su modus vivendi, en cualquier sociedad en la que se encuentren. En otras palabras, las del crítico cultural y periodista Thomas Friedman: «Their own country has become just a place through which other countries and cultures pass» con la norteamericana en primera línea (Friedman: 296).

La producción artística de este «nuevo» grupo inmigratorio ocupa un «espacio sensorial» en el que diferentes gramáticas, representando algunas veces disímiles indicadores culturales, se encuentran entreverados de modo paradójico, y aun opuesto, pero desplegados de manera horizontal o yuxtaposicional. El querer rastrear una genealogía

Myslewsky escribió la reseña para el personal del Bush Theater, recibida por correo electrónico el 8 de marzo, 2005, por el autor de la obra. 
geográfica se problematiza pues es obvio que no se puede hablar de «discontinuidades culturales», o "disonancia cognitiva», entre otras cosas. Las producciones artísticas son constitutivas de un canon alternativo, que reta cualquier lectura simplista o esfuerzo de clasificación en una producción nacional cerrada. Este es el caso del monólogo Nuyor/ Islas, de la autora dominicana, mencionada antes, Chiqui Vicioso, estrenando en el Teatro Las Máscaras en Santo Domingo, el 3 de febrero de 2005, y llevado tres semanas más tarde a Washington Heights, en el Upper Manhattan, para presentarlo ante la comunidad dominicana.

En Nuyor/Islas, ${ }^{10}$ una señora mayor quien vivió en Estados Unidos por más de treinta y cinco años regresa a la República Dominicana, su país de origen, a jubilarse allí. Ocupa una casa en un vecindario nuevo para "Dominicanos ausentes», que han surgido durante las últimas décadas. Aislada del ambiente de su propio «barrio», pues se siente coaccionada a mostrar progreso por todos esos años de trabajo en el extranjero, al moverse a un vecindario mejor, queda completamente sola. Para sentirse acompañada, ve televisión día y noche y habla con ella misma, que es lo que hace durante la representación, motivada, en esta instancia, por el sonido del timbre de la puerta que insinúa la presencia de un cobrador de cuentas. El margen del tiempo-espacio deviene elástico a medida que ella se mueve con facilidad a través de diferentes períodos de su vida. El sentimiento de alienación y desplazamiento es doble, si no triple, al darnos cuenta que la protagonista no se identifica con ninguna comunidad específica. Está realmente atrapada en su propio mundo. Vicioso nos da la oportunidad de ver físicamente este bloqueo al ponerla dentro de una estructura que semeja una caja, con cuatro paredes que se cierran, al final de la pieza, para subrayar esta impresión. En la producción de Santo Domingo, Vicioso puso al interlocutor, con quien se supone que habla la señora durante la presentación, sobre zancos mirando desde el borde de la caja hacia abajo y sus reacciones las vemos proyectadas en una de las paredes interiores de la casa. En Nueva York, se reemplazó por un actor mudo, físicamente sobre el escenario, lo que, creemos, restó un tanto el sentido poético de la presentación al darle un toque realista innecesario.

Entre los tópicos explorados en la pieza se encuentra el del proceso de «proletarización» que muchos latinoamericanos sobrellevan cuando les toca trabajar en fábricas para sobrevivir económicamente. En el caso de la señora mayor, es el único dato de su vida anterior que ella se asegura que trascienda. De manera reiterativa, insiste en que viene de la ciudad de Santiago, en territorio dominicano, "de una buena familia», que tiene clase y alguna educación, porque en su época la única educación que existía para las niñas como ella era "Secretariado Bilingüe», explica, «el que en realidad, no tenía nada que ver con el inglés que se habla en Estados Unidos».

Por otra parte, se da cuenta de sus propios prejuicios, especialmente cuando le toca trabajar con haitianas. Después de aprovechar la oportunidad de ventilar literalmente algunos de los prejuicios que su contexto social ha transferido a la expresión verbal, como, por ejemplo, cuando, por su color oscuro, las compara con Chita, la chimpancé, compañera de Tarzán, o se refiere al supuesto mal olor que exhalan, el que ningún perfume francés podría remediar, añade no sin cierta pesadumbre: «Todo ese bululú sobre los haitianos, y ellos son tan pobres como nosotros, y nosotros somos los haitianos de Nueva York».

10 La mayoría de las citas de esta obra provienen de la representación de la misma en Washington Heights, de la ciudad de Nueva York, en febrero de 2005. Más adelante en el ensayo utilizaré el manuscrito enviado por Vicioso, por correo electrónico. 
Según Judith Butler, es a través de la repetición que uno puede romper con la «historicidad» del «lenguaje del odio» (1997: 37), tal como Vicioso está haciendo aquí, puesto que, la mayoría de las veces, quien musita este lenguaje no es quien lo origina: "The speaker renews the linguistic token of a community, reissuing and reinvigorating such speech. Responsibility is linked with speech as repetition, not as origination» (39).

Esto es especialmente agudo en esta situación puesto que es una encantadora, indefensa y, al parecer, vulnerable señora mayor quien reitera estos prejuicios comunes. En un contexto social en el que hablar mal contra los haitianos es una "práctica ritualizada", ella está simplemente "comunicando lingüísticamente con una historia de hablantes» (Buttler, 1997: 39), como la reacción del público, riendo al reconocer sus propios prejuicios (en la presentación que tuve la oportunidad de asistir en el alto Manhattan), lo hizo evidente.

En vez de los merengues haitianos que sus compañeras de trabajo escuchan, a ella solo le gusta escuchar a Chopin porque esta música mostraría fácilmente que ella es una dama «bien comida, bien vestida y bien educada. No una tiguera marginal». La ironía de esta situación (tal como nos enteramos más tarde en la pieza) es que ella es hija ilegítima de una campesina y del dueño de una fábrica de tabaco, a donde la madre fue a trabajar, y como él solo quería hijos hombres, cuando ella nació las abandonó. El concepto de la «mímica pos-colonial como práctica performativa reflexiva» trabajada por Homi Bhabha (1994), refiriéndose a una posición de subalternidad desde el sitio del enunciado, ayuda aquí a darle sentido a su manía de imitar los aspectos que asocia con las clases altas, lo que a la larga menoscaba su bienestar social, separada como está del resto de la gente de las dos comunidades en las que vivió alguna vez. ${ }^{11}$

Su hija, en quien reconocemos a la autora en su época de estudiante en Nueva York (hasta su descripción física compagina con la misma con el pelo imitando el afro de Angela Davis), trata de hacer reaccionar a la madre:

¡Mamá deja tus «ínfulas»! ¿Tú no te das cuenta de que en los países todos los dominicanos somos negros y que a los gringos les importa un carajo si eres Báez o Vicccini? ¿Que ellos lo que quieren es ganarse sus cuartos y ya? Además siempre asumen que uno es una chusma y se sorprenden cuando te gusta la ópera, o la música clásica, o la comida buena (Vicioso, Nuyor/Islas, Manuscrito, 11).

11 Debemos aclarar aquí que la ubicación del sujeto latino, tal como lo proyectan no pocos estudiosos de la realidad contemporánea del país, como posible «subalterno» en el medio cultural norteamericano, presenta problemas acuciosos. Sin duda, uno de ellos radica, en lo que de manera oportuna señala el dramaturgo Ricardo Bracho, refiriéndose a la dependencia teatral en el dramaturgo más producido del país, Shakespeare, y es «el hecho de que los Estados Unidos se identifica a sí mismo persistentemente, y de manera exclusiva, como una sociedad de colonizadores de proveniencia inglesa» (en Svitch, 2004: 103). Esto hace al individuo latino, por extensión, un subalterno ideal (en el mismo sentido en que los académicos, vía Bhabha y Spivak, sitúan a los hindúes con respecto al imperio británico de donde el concepto del «pos-colonialismo» cobró vida). Sobra decir que la vasta mayoría de los latinos/latinas, sobre todo los provenientes de inmigraciones más recientes, como la que nos ocupa aquí, no se consideran sujetos poscoloniales, puesto que desde su punto de vista, el término también cobijaría a los norteamericanos como colonia que fue del mismo imperio británico. En realidad, cada alma viviente en el continente americano es o desciende de sujetos pos-coloniales. Ahora, una cosa es asociarse a los discursos pos-coloniales desde el punto de vista del Tercer Mundo, hablando académicamente, y otra muy distinta es adoptar conscientemente una entidad "subalterna» con respecto a la sociedad anglo-sajona. No cabe duda que éste sigue siendo un tema de debate entre los representantes de diferentes grupos que constituyen la comunidad. 
La palabra "chusma», como epíteto cualitativo con que la autora describe a una parte de la comunidad vista desde fuera, ha llamado la atención de otros críticos, como José Esteban Muñoz, para quien la palabra «juega con un significado estigmatizador de la identidad Latina dentro y fuera de ambas comunidades» (1999: 181). Refiriéndose a los enclaves hispanos caribeños, Muñoz trata de explicar el término, que no difiere en mucho de la acepción dada por Vicioso en la obra, con el énfasis puesto en las diferencias de clase que la señora trata de afirmar a través de la obra.

[...] Chusmería is a form of behavior that refuses standards of bourgeois comporment. Chusmería is, to a large degree, linked to stigmatized class identity. Within Cuban culture, for instance, being called chusma might be a technique for the middle class to distance itself from the working class. [...] In the United States, the epithet chusma also connotes recent immigration and a general lack of «Americaness» as well as an excessive nationalism $(1999: 182) \cdot{ }^{12}$

La hija muere asesinada, acuchillada por un vulgar ladrón en la esquina de la calle 110 y la avenida 37, en Manhattan, una noche cuando regresaba a su casa del trabajo. Su madre estuvo allí para verla irse, llevándose con ella cualquier esperanza de una vida mejor. Todas las cosas materiales que ella hubiera podido acumular con todas «las tarjetas de crédito, y los planes de venta diferida» perdieron significado. De ahí también el retorno a su país de origen.

No hay duda, como señala la investigadora Rosa Fregoso, que «al re-presentar un continuo cruce de fronteras, el sujeto ‘transfronterizo» opera tanto dentro como fuera de la tradición de las comunidades» (1998: 328), como sucede con los personajes de Vicioso. Otro ejemplo pertinente nos lo brinda el dramaturgo peruano José Castro Urioste, quien vive en Chicago y escribe desde «la ciudad de los vientos».

Su obra Ceviche in Pittsburg se estrenó con el título Trecho de mudez en octubre de 1992, en el teatro The Pitt, en Pittsburgh, PA. Esta pieza pone en escena un encuentro de dos mundos como la conmemoración histórica del evento que la fecha evoca, en la reunión de dos hermanos separados por irreconciliables diferencias desde el momento en que el hermano menor, Toño, vino al mundo desplazando al mayor, Carlos, de la atención familiar. Una vida entera de conflictos no resueltos y de competencia no deseada entre estos dos hermanos parece llegar al punto más crítico durante la visita sorpresa que Carlos le hace a Toño, en Pittsburg, donde el último está haciendo un doctorado en historia peruana. En realidad, la misión de Carlos es darle la noticia de la reciente muerte del padre que ha mantenido en secreto por algún tiempo. No solo la distancia física entre los sitios geográficos en cuestión (los Estados Unidos y el Perú, en donde vive Carlos) hace todavía más grande la brecha entre los dos, sino la ideología vigente en cada lugar también juega un rol importante aquí. Carlos aprovecha el recordar cosas pasadas para cuestionar a Toño la autenticidad de su aprehensión histórica del Perú, el tema de su disertación:

Carlos: [...] Tú vives aquí, con tus libros, tu biblioteca, tus conferencias. Viajas a Londres, a Roma y no sé a dónde más. Y para ti, hermano, para ti el Perú se ha vuelto una noticia

12 Muñoz emplea la palabra «chusma» en su interpretación de la obra Chicas 2000, de la performera cubano-americana Carmelita Tropicana. Por otra parte, y siguiendo con el autor, en Miami, no pocos miembros de las primeras inmigraciones de cubanos llaman «chusma» a las nuevas olas inmigratorias a partir de la llegada de los «marielitos», en 1980, denominados así por haber salido del puerto de Mariel. 
escrita en algún periódico, alguna información que percibes por tu computadora, o un ceviche de fin de semana hecho con pescado de Boston y limones de Pensylvania [...] (1999: 290)

Debemos darle crédito al autor por no haber hecho de Carlos un modelo de virtud. Él es, de acuerdo a Toño, un hombre frustrado quien nunca logró nada por sí mismo, trabajando para su padre en lo que ahora parecen negocios turbios. Mujeriego irredento, a quien su propia mujer dejó por otro hombre cansada de sus infidelidades, trata de seducir a Lucía, la novia de Toño, una periodista de origen colombiano. Lucía funciona, en realidad, como catalizador en ambas situaciones, frenando a Carlos, por un lado, y haciéndole ver a Toño, por el otro, sus puntos débiles.

Lucía: Yo no quiero desaparecer de mi país. Me quedaré unos años más acá terminando mi maestría, pero no me olvido ni quiero que se olviden de mí en Colombia. Allá tú que quieres escribir la historia del Perú en inglés (275).

Su insistencia en escribir para una revista colombiana, aun cuando, según Toño, «su inglés es mejor que el de los gringos y podría conseguir más dinero por su trabajo", hace que Carlos le conteste: "Quizás, no todo es inglés y dinero» (278).

Al final de este tête-à-tête entre los dos hermanos, Toño se reconcilia con su propia soledad, la soledad de su condición híbrida, cuyos efectos no difieren en mucho de la circunstancia anímica en que se encuentra su poco balanceado, y fuera de contexto, hermano Carlos (teniendo en consideración el movimiento feminista de las últimas décadas).

Toño: [...] estás tan solo como yo, que puedo ser el mejor estudiante de historia en los Estados Unidos aunque escriba la historia de un país que empieza a ser un recuerdo para mí, y sigo solo, solo y rodeado de un montón de cartas burocráticas que alaban mi trabajo intelectual. Solos porque nunca aprenderemos a tolerarnos (1999: 299).

Ha habido varias obras en las que la «realidad» de los dos países, sobre todo Estados Unidos y un país latinoamericano, se ha comparado. Este ha sido, de hecho, un leitmotif en el teatro cubano, dentro y fuera de la isla, desde que las dos primeras obras en tratar el tema del exilio irrumpieran en la escena: Alguna cosita que alivie el sufrir (1986), de René Alomá (1947-1987), quien escribía desde Toronto y Week-end en Bahía (1989), de Alberto Pedro (1954-2005), en Cuba, así como explorado en muchas obras que tratan sobre la inmigración latinoamericana (ver Rizk, 2002). Es evidente que en estos casos, en un mundo obviamente dividido y atrincherado en uno u otro lado de la división ideológica prevaleciente durante el período de la Guerra Fría, se daba paso a la percepción o ideología del autor. ${ }^{13}$ La diferencia entre estas obras y la de Castro Urioste es que Carlos y Toño viven en un mundo que ya está bien cimentado en la era de la globalización, en donde uno «tiene que aprender a competir sin barreras afuera de su propio país [...] o aprender a remover las barreras desde dentro» (Friedman, 2000: 230) para poder sobrevivir. En este sentido, en realidad, el individuo ya no tiene ni que salir de su casa, para

13 Casi sobra decir que el hábito de comparar a las dos sociedades no ha desaparecido del teatro latinoamericano o del teatro latino en Estados Unidos como, por ejemplo, lo hace la venezolana Aminta de Lara cuya obra Golondrina tuvo una lectura dramatizada en 2004, en el LATEA, de Nueva York. En la pieza, dos hermanas confrontan su posición ideológica, en contra o a favor de la perspectiva política y la retórica oficial del actual presidente del país, Hugo Chávez, durante el viaje de una de ellas a su país natal. 
sentir los efectos de la globalización, como indica el citado periodista Friedman, «porque [ésta] ha creado un único sitio de mercado [que] puede homogeneizar el consumo simultáneamente en todo el mundo» (278). El problema aparece cuando se pierde el sentido de control de su propia vida y se siente a la merced de fuerzas incontrolables representadas por los «dictadores del mercado», ya sea el horizonte de expectativas de los implacables intelectuales universitarios en la vida de Toño, o los negocios de Carlos en los que la explotación, el fraude y los tratos ilegales parecen ser necesarios para tener éxito.

De acuerdo al investigador chileno José Joaquín Brunner, la «descentralización y desterritorialización» de las culturas, durante la era de la globalización, son dos de las características de la cultura emergente de una «nueva clase de productores y mediadores simbólicos», dado el hecho que ya no son «controlados» desde ningún centro (1990: 44). Por otra parte, no hay duda que a medida que los representantes de las nuevas cifras demográficas se empiezan a mover con desenvoltura dentro de la sociedad receptora no necesariamente quieren reflejar, como hicieron sus antecesores, la problemática tradicional de su comunidad inmediata. Este es el caso del director y dramaturgo venezolano Moisés Kaufman, quien ya se ha forjado un nicho importante en la escena de Broadway y Off-Broadway de Nueva York.

En 1996, Kaufman irrumpe en la escena con la obra Gross Indecency: the Three Trials of Oscar Wilde, sobre el famoso juicio que se le siguió al escritor en Inglaterra, al finalizar el siglo XIX, por «indecente», recibiendo óptimas reseñas y gran aceptación del público. Como es del conocimiento público, Wilde terminó siendo condenado a dos años de trabajos forzados en prisión y murió tan solo tres años después de ser liberado. El crítico Robert Brustein, en su reseña a la obra, señala que la tragedia surge en el momento en que Wilde falla en separar «sus impulsos eróticos del lado estético de su vida», convirtiendo la «moral en arte en una época en que se prefería que el arte fuera una extensión de la moral» ${ }^{14}$ y concluye recordándonos, no sin razón, que: «Even in our more permissive time, when what Wilde called ‘the love that dare not speak its name has grown a little hoarse from shouting it, the Puritan impulse to impose its prohibitionist will on the private lives of citizens remains as insistent as ever».

A finales de 1998, Kaufman y su grupo, Tectonic Theatre Project, decidió ir a Laramie, WY, a investigar la muerte de un estudiante de la Universidad de Wyoming, Matthew Shepard, quien, la noche del 6 de octubre de 1998, fue golpeado brutalmente por dos individuos, amarrado luego a una cerca y dejado a la intemperie, por no otra razón que ser abiertamente gay. Shepard fue encontrado a la mañana siguiente y murió dos días después en un hospital local. El director y su grupo entrevistaron a más de doscientos habitantes del pueblo, material con el que elaboraron la obra, The Laramie Project, que se estrenó en el Denver Center, en el 2000, seguida por una gira nacional en la que recibieron excelentes reseñas. Dos años después el mismo director la llevó a la pantalla grande bajo el mismo título. No son los que perpetraron tan horrendo crimen quienes fueron puestos en el centro del escenario, sino el pueblo entero de Laramie, cual metonimia del país, el que está siendo puesto bajo la mirilla en la obra. En este sentido, la intención del dramaturgo y su grupo no fue solo el tratar de entender un medio que puede producir tan agresivo como injusto comportamiento, sino medir el efecto que tan nefasto crimen tuvo en las vidas de todos los que estuvieron forzados a reconocer públicamente sus prejuicios a través de las entrevistas. Al evitarse también, hasta de manera metafórica, cualquier

\footnotetext{
La traducción de las citas de Brustein del inglés al español son de nuestra autoría.
} 
intento de redención cristiana para los asesinos, se minimizó el sentido de la culpabilidad de los habitantes del pueblo para concentrarse más bien en promover solidaridad con la víctima. Si el "evento", como dijo uno de los entrevistados, "definió al pueblo por un accidente», Kaufman y su grupo permitieron que esta "definición» se quedara con nosotros hasta mucho tiempo después de salir del espectáculo, movidos por la tortura que sufrió Shepard y estremecidos de espanto e impotencia al darnos cuenta de lo bajo que puede caer un ser humano cuando la intolerancia lo corroe.

Desde otra perspectiva que nos acerca al tema de este ensayo, y como hace notar la investigadora Francine Masiello, «crimes of sexuality are valued as a way to confirm the originality of Latin America» (1996: 138), entonces la obra de Kaufman está llamada a trascender esta noción y dirigir la atención al hecho irrefutable de que esta clase de crímenes puede suceder en cualquier parte, aun en el corazón del que pretende ser el país más «democrático» del mundo.

Por otra parte, si tenemos en cuenta las palabras de la investigadora May Joseph cuando señala que: «The expressive stagings of citizenship in the culture of new immigrants enact the need to reinvent community in the interstices of political visibility» (1999: 11), entonces para Kaufman, no hay duda que la «visibilidad política», en vez de estar fundada en las particularidades de una etnicidad propia, está localizada en la urgente como impostergable problemática asociada con los individuos que se identifican como gay en medio de lo que todavía parece ser una sociedad profundamente homofóbica. Ahora, desde la perspectiva de un arte escénico alternativo, Kaufman encaja con ese deslizamiento paradigmático por el que están optando muchos artistas latinos/latinas y latinoamericanos/as quienes están dejando a un lado el concepto y modalidad de «perfoming nación» a favor de "perfoming ciudadanía», en un mundo en el que los movimientos de los derechos humanos parecen ser el último bastión de esperanza para la humanidad.

Para otros teatristas latinos/latinas, «reinventar comunidad» es regresar a la que salieron para explorar los «lugares de la memoria» en oposición a los «sitios de la memoria» (usando el concepto del historiador Pierre Nora, 1997) de sus antepasados. Este es el caso del dramaturgo argentino Mario Diament, quien vive en Miami y hace ya más de una década ha ido estrenando una serie de obras, primero en Miami en inglés, en el New Theater, bajo la dirección de Rafael de Acha y luego en Buenos Aires en español, con las que ha reflexionado sobre la sociedad de la que es originario. ${ }^{15}$ En este sentido, Diament es un perfecto ejemplo del sujeto transnacional en la era posmodernista y globalizada, en que vivimos. Su primera obra que nos llamó la atención fue The Book of Ruth (2000), basada en la vida de una mujer emigró de Polonia a la Argentina a principios del siglo XX, ya estudiada en otra parte (Rizk, 2002). Bástenos agregar aquí que es una pieza un tanto nostálgica como indagatoria de otra inmigración que hizo historia y ayudó a conformar las referencias nacionales del país austral. Estas salen a la superficie a medida que el hijo y la madre se confrontan, ya ella en la tercera edad, en medio de los reproches, sentimientos de culpa y hasta de expiación propia. Pero es Smithereens (2001), ganadora del premio Carbonell 2000, que otorga el gremio anualmente en el Sur de la Florida, en la que nos detendremos aquí. Estrenada en Buenos Aires con el nombre de Esquirlas, la obra presenta un complejo drama entre tres personajes: David, un abogado judío; Sergio, un director de cine de descendencia italiana y Claudia, abogada e hija de un doctor de

15 De hecho, Diament escribe originalmente en español y luego las traduce al inglés. Entrevista con el autor, junio de 2006. 
origen gallego, durante la «guerra sucia» que tuvo lugar en Argentina, de 1976 a 1983, a manos de los militares en el poder. ${ }^{16}$ Es obvio que el origen étnico importa puesto que, de alguna manera, se sugiere que no fueron tratados de la misma manera por los esbirros del poder. Por otra parte, es sabido y mencionado por investigadores, que el segmento judío fue visto como potencial sospechoso opositor, debido a su notoria militancia en los partidos socialistas y anarquistas en la primera mitad del siglo XX, por encima de otros grupos, lo que, de paso, el mismo David reconoce en la obra, cuando Sergio le reclama el haberse ido a Israel:

Sergio: Pero, ¿por qué a Israel?... Vos eras el primero en burlarte de todo lo que sonaba judío...

David: A lo mejor fue por eso... Admitamos llamarse D'Alessandro en Argentina siempre fue más fácil que llamarse Rabinovich. No sé... Creo que sentí la necesidad de estar en un lugar donde llamarse Rabinovich fuera lo normal... (Manuscrito, enviado por el autor por correo electrónico, 10).

La acción oscila entre 1990, cuando tiene lugar el re-encuentro de los personajes, y los años setenta cuando sucede la acción. La «memoria» de lo que pasó desde el momento en que Claudia, pareja de Sergio, fue enviada por éste al encuentro de David, su mejor amigo, quien, de manera previsible, se enamoró de ella, es de lo que trata la obra. Claudia, abogada de derechos humanos, bien pronto se vio comprometida por su ocupación y pasó a ser sospechosa de beligerancia en grado sumo. Cuando el mundo se fue cerrando alrededor de ellos, David, quien vivía con Claudia, decide exilarse en Israel, y Sergio, pensando que si la entregaba a un militar conocido, con la promesa de que no la iba a matar, y a cambio también del financiamiento de sus películas, quedaba a salvo, la delata para hundirse después de su vil acción en un marasmo mental que lo conduce al alcoholismo.

En realidad, la obra es un compendio de muchos temas: en primera instancia, es una recreación "realista», hasta donde el término alcanza, de lo que sucedió no solo en el país entero sino dentro y fuera de los antros en donde se llevaron a cabo las torturas a través de las que sufrió Claudia. Claro está, si por "realismo» entendemos el énfasis puesto en la proximidad del espectador ante la intimidad física de las descripciones de las sesiones de tortura por las que pasó la protagonista. Aquí lo de «sucio", que califica a esta guerra, deja de ser metáfora para convertirse en horrenda realidad, pues solo así se puede calificar la conducta de algunos de los personajes de la obra, aunque el "hedor» y el sentimiento de culpabilidad alcanza a muchos. En este sentido, Sergio no puede ser más claro:

¡Despabilate, Rabinovich! Andá, salí a caminar un poco por la calle... Mirale la cara a la gente... Nadie te mira a los ojos... No hay más inocentes... Todos somos culpables... Todos fuimos cómplices... ¿Vos creés que un grupo de delirantes en uniforme puede matar 30.000 personas si la gente no se lo permite? No, hermanito... Pudieron hacerlo porque los dejamos hacer... Es tan simple como eso... Los dejamos hacer... Vos y yo... (88).

16 De manera hipotética por supuesto, nos preguntamos si este énfasis en la diversidad étnica del país del Cono Sur, lo que no sucede hasta donde nuestros conocimientos alcanzan en la dramaturgia que se ha desarrollado alrededor del tema de la opresión militar, no será un reflejo de la constante problemática étnica-racial que aqueja la sociedad en la que vive el propio autor. 
Por otra parte, desde el punto de vista histórico, y teatralmente hablando, la obra marca el fin de una época encarnado en el personaje del padre de David, quien se erige en el último actor de la última pieza en iddish, que tuvo en Buenos Aires gran apogeo durante las primeras tres décadas del siglo XX (ver Rizk, 2005), cuyo espectador final resulta ser la misma Claudia, quien acude a verlo para poder comparar «infiernos» con un sobreviviente de los campos de concentración alemanes. Por esta vía, la obra es también un llamado a la tolerancia y al mismo tiempo una denuncia al racismo de una sociedad que abrazó la filosofía nazista, la que sale a la superficie de vez en cuando aun a nivel popular. La sombra del Tercer Reich nunca está muy lejos; de hecho, Claudia se refiere a Sergio como la «Leni Riefenstahl de la Argentina» (89), haciendo referencia a los documentales que hizo la directora alemana a raíz de los juegos olímpicos de 1936 cuyo efecto, de paso, se equipara con la copa mundial de fútbol que tuvo lugar en el país durante la dictadura. En este sentido, la obra se conecta con un buen número de dramaturgos, escritores y cineastas norteamericanos que han profundizado sobre esta temática y mantenido viva la «memoria» del holocausto.

En cuanto a América Latina, la pieza nos trae a la mente La muerte y la doncella, del autor chileno Ariel Dorfman, sobre el encuentro posterior de un torturador con su posible víctima durante los peores años de la dictadura militar iniciada por Augusto Pinochet a partir de 1973. Nacido en Argentina, Dorfman ha vivido en Estados Unidos desde la llegada de los militares al poder en Chile, país en donde se crió. Después del estreno inicial de la obra, sin pena ni gloria, en Santiago, en 1991, se presentó primero en el teatro Royal Court Upstairs de Londres, y luego de recibir el premio London Time Out por la mejor obra del año se mudó al Royal Court Mainstage. En marzo de 1992 se estrenó en Broadway, bajo la dirección de Mike Nichols y teniendo en el reparto a Glenn Close (quien ganó el «Tony» por su actuación), Richard Dreyfuss y Gene Hackman. ${ }^{17}$ La crítica en general fue benevolente y aunque reconoció la importancia del tema no se interesó mucho por una experiencia que el público norteamericano, en ese entonces, no reconocía como propia. Ahora lo que sí creó la obra fue una marcada controversia por parte de grupos hispanos interesados que protestaron vehementemente por la no inclusión de actores de origen latino en el reparto. Esta fue una de las primeras instancias que las voces discrepantes de la comunidad se hicieron sentir asegurando su participación en futuros proyectos que le atañen directamente. Este, por supuesto, no ha sido el caso en un Miami más equitativamente multi-cultural, en términos sociales, en donde la mayoría de los personajes latinoamericanos creados por Diament han sido representados por actores de origen latino. Su última obra hasta la fecha, Blind Date, en la que pone en la escena a un escritor invidente, con todas las características de un José Luis Borges ya mayor, estrenó en 2003 en Miami y un año más tarde fue llevada a la escena por Carlos Ianni, en Buenos Aires, bajo el título Cita a ciegas. En 2005 obtuvo el premio «María Guerrero" como la mejor obra argentina estrenada del año.

No hay duda que en todos los casos de los autores expuestos aquí, el «nivel transnacional de integración» ya no obedece, como bien hace notar Gustavo Lins Ribeiro refiriéndose a este tipo de experiencia, "a la lógica clasificatoria de inclusividad como

17 En 1994, la obra fue llevada al cine por Roman Polanski con los actores Sigourney Weaver, Ben Kingsley y Stuart Wilson. En cuanto a la producción dramática de Dorfman, tenemos noticias de Widows, adaptación de su novela homónima (escrita en 1983), presentada en varias ciudades de Estados Unidos, entre ellas Dallas, Boston y Nueva York; así como The Other Side (2006) y Purgatorio (2006). 
sucede en otros niveles» (1988: 327). Sus obras atraviesan, en este sentido y como esperamos haber demostrado aquí, diferentes niveles de integración desde un sentido yuxtaposicional, proyectándose, de hecho, hacia un transnacionalismo que, como advierte el citado investigador, "es imposible de relacionar con un territorio circunscrito" de manera exclusiva.

\section{REFERENCIAS}

Acosta-Belén, Edna y Barbara R. Sjostrom (eds.). (1991). The Hispanic Experience in the United States: Contemporary Issues and Perspectives. Nueva York: Praeger.

Alomá, René. (1992). Alguna cosita que alivie el sufrir, Teatro cubano contemporáneo. Trad. de Alberto Sarraín. Ed. Carlos Espinosa Domínguez. Madrid: Centro de Documentación Teatral.

Anzaldúa, Gloria. (1987). Borderlands/La Frontera. The New Mestiza. San Francisco: Aunt Lute.

Aparicio, Frances R. y Susana Chávez-Silverman (eds.). (1997). Tropicalizations: Transcultural Representations of Latinidad. Hanover/Londres: University Press of New England.

Bhabha, Homi K. (1990). The Other Question, Difference, Discrimination, and the Discourse of Colonialism. En Russell Ferguson, Martha Gever, Trinh T. Minh-ha y Cornel West (eds.). Out There: Marginalization and Contemporary Cultures. Cambridge: MIT Press.

-. (1994). The Location of Culture. Londres: Routledge.

Brunner, José Joaquín. (1990). Tradicionalismo y modernidad en la cultura Latinoamericana. En Documento de Trabajo-Serie Educación y Cultura (Chile). FLACSO 4: 1-44.

Brustein, Robert. (2000). The New Republic, quoted by the Gross Indecency: the Three Trials of Oscar Wilde. Obtenido desde <http://www.law.umkc.edu/faculty/projects/ ftrials/wilde/grossindecency.html>.

Butler, Judith. (1997). Excitable Speech: A Politics of the Performative. Nueva York: Routledge.

Castro Urioste, José. (1999). Ceviche en Pittsburg, Dramaturgia Peruana. José Castro Urioste y Roberto Ángeles (eds.). Lima/Berkeley: Centro de Estudios Literarios Antonio Cornejo Polar/Latinoamérica Editores. 269-299.

Chávez-Silverman, Susana. (2003). Gendered Bodies and Borders in Contemporary Chican@ Performance and Literature. En Alicia Gaspar de Alba (ed.). Velvet Barrios: Popular Culture \&o Chicana/o Sexualities. 215-27. Nueva York: Palgrave McMillan.

Colecchia, Francesca M y Luis F. González-Cruz (eds.). (1992). Cuban Theater in the United States: A Critical Anthology. Tempe: Bilingual Press/Editorial Bilingüe.

Cortina, Rodolfo J. (ed). (1991). Cuban American Theater. Houston: Arte Público Press.

De la Campa, Román. (2005). Hispanism and its Lines of Flight. En Mabel Moraña (ed.). Ideologies of Hispanism. Hispanic Issues 30. 300-10. Nashville: Vanderbilt University Press. 
Escarpanter, José. (1988). «El teatro cubano fuera de la isla». En Escenario de dos mundos: Inventario teatral de Iberoamérica. Tomo 1. Madrid: Centro de Documentación Teatral/Ministerio de Cultura.

Fregoso, Rosa Linda. (1998). Sacando los trapos al sol in Lourdes Portillo's Melodocumystery, "The Devil Never Sleeps». En Diana Robin e Ira Jeffe (eds.). Redirecting the Gaze: Gender, Theory, and Cinema in the Third World. 307-29. Albany: State University of New York Press.

Friedman, Thomas L. (2000). The Lexus and the Olive Tree. Nueva York: Anchor Books.

Horno-Delgado, Asunción, Eliana Ortega, Nina M. Scott y Nancy Saporta Sternbach (eds.). (1989). Breaking Boundaries. Latina Writings and Critical /Readings. Amherst: University of Massachusetts Press.

Huerta, Jorge. (1982). Chicano Theatre: Themes and Forms. Ypsilandi, Michigan: Bilingual Press/Editorial Bilingüe.

-. (2001). Chicano Drama: Performance, Society and Myth. Nueva York: Cambridge University Press.

Jiménez-Muñoz, Gladys M. (1993). The Elusive Signs of African-Ness: Race and Representation Among Latinas in the United States. En Border/Lines 29/30: 9-15.

Joseph, May. (1999). Nomadic Identities: The Performance of Citizenship. Minneapolis: The University of Minnesota Press.

Kanellos, Nicolás. (1983). Mexican American Theatre: Then and Now. Houston: Arte Público.

-. (1990). The History of Hispanic Theater in the United States: Origins to 1940. Austin: University of Texas Press.

Lins Ribeiro, Gustavo. (1998). Cybercultural Politics: Political Activism at a Distance in a Transnational World. En Sonia E. Álvarez, Evelina Dagnino y Arturo Escobar (eds.). Cultures of Politics / Politics of Cultures: Re-Visioning Latin American Social Movements. 325-52. Boulder: Westview Press.

Manzor, Lilian y Alberto Sarraín (eds.). (2005). Teatro cubano actual: Dramaturgia escrita en Estados Unidos. La Habana: Edición Alarcos.

Masiello, Francine. (1996). Melodrama, Sex, and Nation in Latin America's Fin de Siglo. En Doris Sommer (ed.). The Places of History Regionalism Revisited in Latin America. 134-43. Durham/Londres: Duke University Press.

Muñoz, José Esteban. (1999). Disidentifications: Queers of Color and the Performance of Politics. Minneapolis: University of Minnesota Press.

Nora, Pierre. (1997). Les Lieux de Mémoire. París: Gallimard.

Ott, Gustavo. (1999). 80 dientes, 4 metros y 200 kilos. Madrid: Ediciones Cultura Hispánica.

-. (2003). Tu ternura Molotov. Santander: Ayuntamiento de Santander.

Pedro Torriente, Alberto. (1989). Week-End en Bahía, Primer Acto. 228: 84-105.

Portes, Alejandro y Kelly Hoffman. (2003). Latin American Class Structures: Their Composition and Change during the Neoliberal Era. Latin American Research Review 38.1: 41-82.

Ramos-Perea, Roberto (ed.). (2006). Boletín del Archivo Nacional de Teatro y Cine del Ateneo Puertorriqueño 5 (enero a julio).

Rizk, Beatriz J. (2000). Miami: meca del exilio cubano y escenario medido aunque efervescente. Latin American Theatre Review Vol. 34, 1: 229-45. 
-. (2002). Teatro y diáspora: Testimonios escénicos latinoamericanos. Irvine, CA: Ediciones de Gestos.

-. (2005). La dramaturgia judeo-argentina: Cien años de manifiesta presencia en el país austral. Theatralia VII: 123-36.

Romero, Mary, Pierrette Hondagneu-Sotelo y Wilma Ortiz (eds.). (1997). Challenging Fronteras: Structuring Latina and Latino Lives in the U.S. Nueva York: Routledge.

Rosales, Arturo F. (1997). Chicano: The History of the Mexican American Civil Rights Movement. Houston: Arte Público Press.

Shohat, Ella y Robert Stam. (1994). Unthinking Eurocentrism: Multiculturalism and the Media. Nueva York: Routledge.

Spivak, Gayatri Chakravorty. (1996). The Spivak Reader: Selected Works. Donna Landry and Gerald Maclean (eds.). Londres/Nueva York: Routledge.

Svich, Caridad. (2000). Home, Desire, Memory: There Are no Borders Here. En Alberto Sandoval-Sánchez y Nancy Saporta Sternbach (eds.). Puro Teatro: A Latina Anthology. 319-24. Tucson: University of Arizona Press.

- (2004). Re-Shaping the Future: Afterthoughts on a Community Roundtable. The Journal of American Drama and Theatre Vol. 16, 3: 97-109.

Tubert, Susana. (2000). Tales of a South-of-the-Border / North-of-the-Stereotype Theater Director. En Alberto Sandoval-Sánchez y Nancy Sapora Sternbach (eds.). Puro Teatro: A Latina Anthology. 325-329. Tucson: University of Arizona Press. 